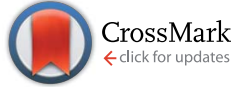

Cite this: RSC Adv., 2017, 7, 17208

Received 26th December 2016 Accepted 9th March 2017

DOI: 10.1039/c6ra28721b

rsc.li/rsc-advances

\title{
A simple 3D cryogel co-culture system used to study the role of CAFs in EMT of MDA-MB-231 cells $\dagger$
}

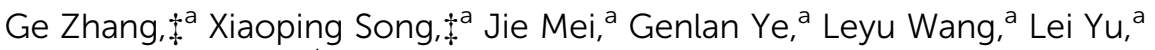 \\ Malcolm M. Q. Xing ${ }^{b}$ and Xiaozhong Qiu ${ }^{\star a}$
}

Breast cancer is one of the most common epithelial tumors and can seriously endanger women's health. Studies have shown that carcinoma-associated fibroblasts (CAFs) possess myofibroblast features and play an important role in the growth and metastasis of breast cancer. Because of the lack of an applicable animal model, the exact role of CAFs in tumor metastasis is still unclear. In this research, a simple three-dimensional (3D) cryogel co-culture system was developed to evaluate the role of CAFs in breast cancer metastasis. It was found that CAFs could promote epithelial-mesenchymal transition of MDA-MB-231 cells (human breast cancer cells) in a 3D cryogel co-culture system in vitro. Then the coculture human breast cancer system was used to construct a xenograft tumor mouse model in vivo. It was found that CAFs could promote deterioration and metastasis of MDA-MB-231 cells in a xenograft tumor mouse model in vivo.

\section{Introduction}

Patients suffering from breast cancer often die of malignant metastases rather than the primary cancer. The local microenvironment, or niche, of a breast cancer cell plays an important role in the cancer development. ${ }^{1}$ A major component of the niche is the extracellular matrix (ECM), which is a complex network of macromolecules including diffusible growth factors, cell factors, and fibroblast secretions. ${ }^{2}$ The ECM is related to the fibroblast secretions and the fibroblasts combine to form a stromal layer for the breast cancer cells to settle in. ${ }^{3}$

In pathological conditions, fibroblasts form the largest number of matrix cells in human breast cancer. The ECM secreted by the fibroblasts provides support, nutrition, regulatory molecules, as well as a variety of physical and chemical conditions for the growth, invasion and metastasis of the tumor cells. ${ }^{4}$ A previous study showed that carcinoma-associated fibroblasts (CAFs) possessed myofibroblast features. ${ }^{5}$ CAFs can secrete tumor growth factors to promote tumor cell growth. They can also raise the endothelial progenitor cells into tumor tissue to promote the blood vessel formation in vivo. ${ }^{6}$ The

\footnotetext{
${ }^{a}$ Department of Anatomy, Guangdong Provincial Key Laboratory of Construction and Detection in Tissue Engineering, Southern Medical University, Guangdong, Guangzhou 510515, China. E-mail: qqiuxzh@163.com

${ }^{b}$ Department of Mechanical Engineering, Manitoba Institute of Child Health, University of Manitoba, Winnipeg, MB R3T 2N2, Canada

$\dagger$ Electronic supplementary information (ESI) available. See DOI: $10.1039 / \mathrm{c} 6 \mathrm{ra} 28721 \mathrm{~b}$

\$ Equal contribution.
}

volume and weight of harvested tumors from the mere transplantation of MCF-7 cells were smaller than those from the transplantation of the co-culture cells including MCF-7 cells and CAFs in subcutaneous tumor mouse model. ${ }^{3}$ Previous research of co-culturing always used an indirect method, which cultured the breast cancer cells with the CAF-cultured medium or directly mixed the secretive factors of CAFs in the medium of cancer cells. ${ }^{7,8}$ However the direct effect of CAFs on the epithelial-mesenchymal transition (EMT) of tumor cells is still unclear.

As for a desirable biomaterial, it should provide a proper microenvironment parallel to the in vivo situation for the surrounding cells, and thus contribute to further study on the behavior of cells. ${ }^{9}$ During the past few years, cryogels have been applied widely in tissue engineering and biomedical fields, which was related to their inherent interconnected porous structures and ease of formation in comparison to other macropore forming techniques. ${ }^{10,11}$ Cryogels are porous biomaterials obtained by forming hydrogels below the melting point of a solvent. Gelatin modified with pendant methacrylate groups (GelMA) derived cryogel is a type of cell and tissue compatible biomaterial and its porous structures could increase cell trafficking, angiogenesis and elasticity. ${ }^{12}$ In addition, the gelatin which contains inherent cell adhesive peptide sequences instead of the traditional biomaterials linked with extra cell adhesive peptides had some useful advantages, such as costeffectiveness, safety and lack of immunogenicity. ${ }^{13}$

Because of the existence of the macroporous structures, the unmodified cryogels often suffered from lack of mechanical strength and were always limited in application. ${ }^{14}$ Nevertheless, 
with for breast cancer, the high elasticity of the cryogels enabled it to act more like human mammary gland tissues for imitating the tumor microenvironment compared with other traditional stiffer materials. Inspired by this, it was assumed that the elastic GelMA cryogel scaffold could be used as an ideal platform to study the mechanism of breast cancer transfer in vitro. Additionally, GelMA cryogels are easily acquired and cause no environmental harm. A GelMA cryogel could also be produced in any shape. ${ }^{12}$ Porous structures in the GelMA cryogel facilitated blood vessel formation in vivo, ${ }^{\mathbf{1 5 , 1 6}}$ which was important in tumorigenesis. As our initial scanning electron microscopy (SEM) has shown that some modification of the GelMA cryogel could reduce the pore size and the seeded cells could cover the porous structures. ${ }^{17}$ The macroporous structure of the GelMA cryogel is both positive and negative, because it might be beneficial for the breast cancer cells to adhere along the wall of the microporous structure and but can also cause them to grow intensively inside the cryogel. Under the condition of threedimensional (3D) co-culture system, the joint structure between the seeding cells and the adhered wall of the cryogel pores could become thick and tenacious. Here, the CAFs seeded on the GelMA cryogel were used to form a 3D stromal cell layer for the 3D culture of MDA-MB-231 breast cancer cells. This simple 3D cryogel co-culture system was a useful tool for studying the role of the CAFs in the metastasis of breast cancer cells in vitro and in vivo.

\section{Experimental section}

\section{Patients and tumors}

The study involving human samples was approved by ethical principles obtained from the Human Research Ethics Committee (HREC). Samples were all collected from the Zhujiang Hospital of the Southern Medical University (Guangzhou, China). Before samples were collected, informed consent was obtained from patients after full explanation of the purpose and nature of all procedures. The guidelines followed in our experiments with live subjects had been approved and supervised by the Medical Ethics Committee of Nanfang Hospital of the Southern Medical University in 2015. This study has satisfied the relevant guidelines of the Medical Ethics Committee.

\section{Mice}

The nude mice (female, 4-5 weeks) were purchased from the Experimental Animal Department of the Southern Medical University. A permit for using the mice was given by the Institutional Animal Care and Use Committee (IACUC) of the Southern Medical University.

\section{Materials}

Gelatin, tetramethylethylenediamine (TEMED), and ammonium persulfate (APS) were obtained from Sigma-Aldrich (USA). $N, N^{\prime}$-Methylene-bis-acrylamide was purchased from Sigma-Aldrich (USA). The live/dead cell staining kit was Molecular Probes ${ }^{\mathrm{TM}}$ (Thermo Fisher Scientific, USA). All of the primary antibodies were purchased from Abcam (UK). Alexa
Fluor ${ }^{\circledR} 488$ Donkey Anti-Rabbit IgG $(\mathrm{H}+\mathrm{L})$ and Alexa Fluor ${ }^{\circledR}$ 568 Donkey Anti-Mouse IgG $(\mathrm{H}+\mathrm{L})$ were from Life Technologies (USA).

\section{Gelatin cryogel preparation}

As previous reports described, ${ }^{12}$ GelMA was synthesized as follows: gelatin solution was obtained by dissolving $10 \%(\mathrm{w} / \mathrm{v})$ type A porcine skin gelatin (Sigma-Aldrich) in stirred Dulbecco's phosphate buffered saline (DPBS; Gibco) at $50{ }^{\circ} \mathrm{C}$ for $1 \mathrm{~h}$. Methacrylic anhydride (Sigma-Aldrich) was then added dropwise to give a final volume ratio of $1: 4$ methacrylic anhydride : gelatin solution. The mixture was stirred at $50{ }^{\circ} \mathrm{C}$ for $1 \mathrm{~h}$. The resulting mixture was dialyzed in 12-14 kDa molecular weight cutoff tubing (Spectrum Labs) for four days against distilled water $\left(\mathrm{dH}_{2} \mathrm{O}\right)$, and the water was replaced frequently. The dialyzed solution was lyophilized, and the resulting GelMA was stored at $-20{ }^{\circ} \mathrm{C}$ until use. For the synthesis of the cryogels, the dissolved GelMA was mixed with $0.5 \%(\mathrm{w} / \mathrm{v})$ APS and $0.1 \%(\mathrm{w} / \mathrm{v})$ TEMED. The mixture was immediately pipetted into $2 \mathrm{~mm} \times 9 \mathrm{~mm}$ circular dies and placed in a freezer at $-80{ }^{\circ} \mathrm{C}$ for $12 \mathrm{~h}$. The resulting cryogels were then thawed and hydrated in $\mathrm{dH}_{2} \mathrm{O}$. The cryogels were thoroughly washed three times with sterilized DPBS solution to remove and chemical residues. Then the cryogels were immersed in $75 \%$ ethyl alcohol overnight, and then thoroughly washed several times with sterilized DPBS solution to remove the remaining ethyl alcohol. The sterilized cryogels were immersed in cell culture medium prior to use.

\section{Isolation of CAFs and cell culture}

CAFs were sourced from human breast para-carcinoma tissue specimens. Tissue samples were maintained in F-12 medium (Gibco) supplemented with 10\% fetal bovine serum (FBS; Gibco). The tumor was dissociated enzymatically into a single cell suspension. After being cultured for $0.5 \mathrm{~h}$, the non-adherent cells were removed. The adherent cells were then cultured in F12 medium containing 10\% FBS, $100 \mathrm{U} \mathrm{ml}^{-1}$ penicillin (Sigma) and $100 \mu \mathrm{g} \mathrm{\textrm {L } ^ { - 1 }}$ streptomycin (Sigma) at $37{ }^{\circ} \mathrm{C}$ in $5 \%$ carbon dioxide. The MCF-7 and MDA-MB-231 cells were cultured in high glucose Dulbecco's modified Eagle's medium (Gibco) at the same conditions.

For cell seeding in the cryogels, $200 \mu \mathrm{l}$ of a cell suspension containing $10^{7}$ cells per ml of CAFs was dropped onto the cryogel. Then the cryogel was cultured with F-12 medium in a sixwell plate. After culturing for three days, the cryogels were seeded with MDA-MB-231 cells $\left(10^{7}\right.$ cells per $\left.\mathrm{ml}\right)$ and the cultured in F-12/high glucose mixed medium (3D-MDA/CAF group). The same density of MDA-MB-231 cells were seeded on pure cryogels and cultured in high glucose medium (3DMDA group). The MDA-MB-231 cells cultured in a twodimensional (2D) plate were called the 2D group.

\section{Scanning electron microscopy (SEM)}

The samples were washed three times ( 5 min each) with DPBS, fixed in $10 \%$ glutaraldehyde overnight, and then rinsed three times (5 min each) with DPBS. Then the fixed samples were 
dehydrated using an ethanol series with ascending concentrations of $50 \%, 60 \%, 70 \%, 80 \%, 90 \%, 95 \%$ for $5 \mathrm{~min}$ and $100 \%$ (twice for $5 \mathrm{~min}$ ), and then air dried afterwards. The samples were cut into ultrathin sections $(\sim 50 \mathrm{~nm})$, stained with uranyl acetate and lead citrate, and then photographed using a S3000N SEM (Hitachi, Japan).

\section{Live/dead staining assay}

Cyto-compatibility of the cryogels and the viabilities of different cells including MCF-7 cells, MDA-MB-231 cells and CAFs cultured in the gel were determined separately using the live/ dead staining assay. After culturing for three days, $100 \mu \mathrm{l}$ of the live/dead staining reagent was added to each gel which was then incubated for $30 \mathrm{~min}$ at $37^{\circ} \mathrm{C}$, shielded from light. The stained samples were photographed using an IX fluorescence microscope (Olympus). The status of cell survival was defined as the percentage ratio of live/dead cells, where calcium-acetoxymethyl (AM) green fluorescence stained cells were live, and ethidium homodimer-1 red fluorescence stained cells were dead.

\section{Scratch wound healing assay}

The effects of CAFs on MDA-MB-231 cell migration in the 3D cryogel co-culture system were examined using the scratch wound healing assay. The samples were divided into three groups: the 3D-MDA/CAF group, the 3D-MDA group and $2 \mathrm{D}$ pure culture groups. After culturing for three days, the cells from the three groups were dissociated enzymatically and then collected. Cells $\left(10^{5}\right)$ were seeded onto $60 \mathrm{~mm}$ cell culture dishes. After the cells reached approximately $90 \%$ confluence as a monolayer, the surface of each dish was gently and slowly scratched with three collateral lines, using a disinfected $10 \mu \mathrm{l}$ pipette tip. Photographs of the scratch wound healing areas were obtained at $0 \mathrm{~h}, 6 \mathrm{~h}, 12 \mathrm{~h}$ and $24 \mathrm{~h}$ from the same area. The closing rate was calculated using the following formula:

$$
\text { Closing rate }=\frac{\text { cell area }}{\text { vision area }}
$$

The vision area is the number of pixels of the whole scratch wound vision. The cell area is the number of pixels of the scratch wound area that the migratory cells covered in that vision.

\section{Immunofluorescence staining}

After culturing for three days, the samples of the 3D-MDA/CAF group, 3D-MDA group and 2D group were washed with DPBS and fixed overnight at $4{ }^{\circ} \mathrm{C}$ using $4 \%$ paraformaldehyde. The embedded samples were cut into $5 \mu \mathrm{m}$ thick sections at $-20{ }^{\circ} \mathrm{C}$ in a CM1950 cryostat (Leica) and dried at $40{ }^{\circ} \mathrm{C}$ for $4 \mathrm{~h}$. The samples were then washed with DPBS and permeabilized with $0.2 \%$ Triton $\mathrm{X}-100$ at room temperature for $20 \mathrm{~min}$. After blocking with $1 \%$ bovine serum albumin/DPBS at room temperature for $30 \mathrm{~min}$, the samples were incubated overnight at $4{ }^{\circ} \mathrm{C}$ with primary antibodies. The primary antibodies used were: mouse Anti-Ki67 antibody (1 : 100, Abcam), rabbit anti-E cadherin antibody ( $1: 500$, Abcam), mouse anti-N cadherin antibody ( $1: 1000$, Abcam), rabbit anti-fibroblast activation protein (FAP) antibody (1:1000, Abcam), and rabbit anti- $\alpha$ smooth muscle actin ( $\alpha \mathrm{SMA})$ antibody $(1: 100$, Abcam). Then samples were washed three times with DPBS followed by incubation with the secondary antibodies: Alexa Fluor ${ }^{\circledR} 568$ donkey anti-rabbit IgG $(\mathrm{H}+\mathrm{L})(1: 500)$ and Alexa Fluor ${ }^{\circledR} 568$ donkey anti-mouse $\operatorname{IgG}(\mathrm{H}+\mathrm{L})(1: 500)$ for $1 \mathrm{~h}$. The samples were rinsed with DPBS, and then further stained with $4^{\prime}, 6$-diamidino-2phenylindole (DAPI) for $1 \mathrm{~h}$ and then imaged using a confocal microscope.

\section{Western blot analysis}

After culturing for three days, the samples were washed three times with DPBS and then shattered with liquid nitrogen. The total protein content of all the samples was determined using a radio immunoprecipitation assay protein extraction solution. The total protein concentrations were determined using a bicinchoninic acid (BCA) protein assay (KeyGEN). The proteins $(20 \mu \mathrm{g}$ per lane) were separated using $12 \%$ sodium dodecyl sulfate - polyacrylamide gel electrophoresis (SDS-PAGE) and then transferred electrophoretically to poly(vinylidene difluoride) membranes. The membranes were blocked for $2 \mathrm{~h}$ at room temperature in 5\% (w/v) skimmed milk, and then incubated with antibodies against rabbit anti-glyceraldehyde 3phosphate dehydrogenase (GAPDH; 1:1000), mouse antivimentin $(1: 100)$, rabbit anti-E cadherin $(1: 1000)$ and mouse anti-N cadherin $(1: 500)$ at $4{ }^{\circ} \mathrm{C}$ overnight. After incubation with the primary antibody, the membranes were washed three times with Tris buffered saline buffer and then incubated with the appropriate horseradish peroxidase-linked secondary antibodies for $1 \mathrm{~h}$ at room temperature. The optical density was determined using a supersignal chemiluminescent substrate (Santa Cruz) according to the manufacturer's instructions.

\section{Animal experiments}

Female nude mice (4-5 weeks old, $18 \pm 2 \mathrm{~g}$ ) were anesthetized and divided into three groups (the $3 \mathrm{D}-\mathrm{MDA} / \mathrm{CAF}$ group, the $3 \mathrm{D}$ MDA group and the 2D group) with six mice per group. For the 3D-MDA/CAF group, the hybrid, which was MDA-MB-231 cells co-cultured with CAFs in the 3D cryogel scaffold, was cultured for three days and then implanted into the left axilla of a mouse. For the 3D-MDA group, the hybrid, which was pure MDA-MB231 cells cultured in the $3 \mathrm{D}$ cryogel scaffold without the CAF stromal cell layer, was cultured for three days and then implanted into the left axilla of a mouse. In the 2D group, the pure MDA-MB-231 cells were injected subcutaneously into the left axilla of the mouse. All the mice of the three groups were raised for seven weeks and then sacrifice. There were no deaths in any of the three groups before the sacrificing. The tumorigenicity of the nude mice is shown in Table S1 (ESI†).

\section{Hematoxylin-eosin staining}

After seven weeks, post-transplant, the animals were sacrificed, and the tumor tissues were harvested. The samples were then rinsed with DPBS, fixed overnight at $4{ }^{\circ} \mathrm{C}$ with $4 \%$ 
paraformaldehyde, and then cut into $5 \mu \mathrm{m}$ thick slices. The sections were stained with hematoxylin-eosin (H\&E) and then photographed under an optical microscope.

\section{Statistical analysis}

Data are presented as means \pm standard deviations. Statistical analysis was performed using SPSS 13.0 software. Differences among the groups were assessed using one-way analysis of variance (ANOVA). A value of $p<0.05$ or $p<0.01$ was considered to be statistically significant.

\section{Results and discussion}

\section{Isolation of CAFs from clinical human breast cancer samples}

The "seed and soil" hypothesis presupposes that it is necessary to offer a proper host microenvironment (the soil) for optimal growth of tumor cells (the seed). ${ }^{18}$ The tumor microenvironment is the cellular environment in which the tumor exists, including the surrounding blood vessels, immune cells, fibroblasts, bone marrow derived inflammatory cells, lymphocytes, signaling molecules and the ECM. ${ }^{19}$ As for the establishment of cancer models, researchers have demonstrated that $3 \mathrm{D}$ scaffold materials had many advantages when used in co-cultures. The cancer cells seeded on 3D scaffolds displayed malignant properties because of the increasing expression of the genes involved in initiation, progression, and colonization of metastasis. ${ }^{20}$ In addition, 3D materials promoted the selection, growth and enrichment of cancer cells. Tumor cells provided the cell-cell and cell-matrix interaction microenvironment in 3D scaffolds. Compared with that of the $2 \mathrm{D}$ culture, the microenvironment of the $3 \mathrm{D}$ co-culture system was more similar to the tumor niche in vivo. ${ }^{21,22}$ CAFs are the most common component of the tumor

stroma, especially in breast and pancreatic cancers. ${ }^{23,24}$ Previous research on co-culturing always used an indirect method which cultured the breast cancer cells with the CAF-cultured medium or mixed the secretive factors of CAFs directly in the medium of the cancer cells. ${ }^{7,8}$ However, as the mechanisms which influence

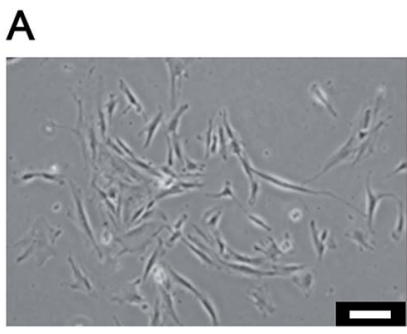

DAY 3

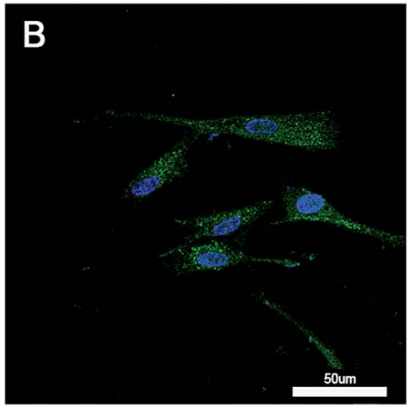

Fig. 1 Identification of CAFs. (A) The morphology of separated CAFs after culturing for three and seven days shown under light microscopy (scale bar $=100 \mu \mathrm{m}$ ). (B) Immunofluorescence assay of the CAFs after culturing for three days. Blue: DAPI, green: FAP. (C) Immunofluorescence assay of the CAFs after culturing for three days. Blue: DAPI, Green: $\alpha-S M A$.

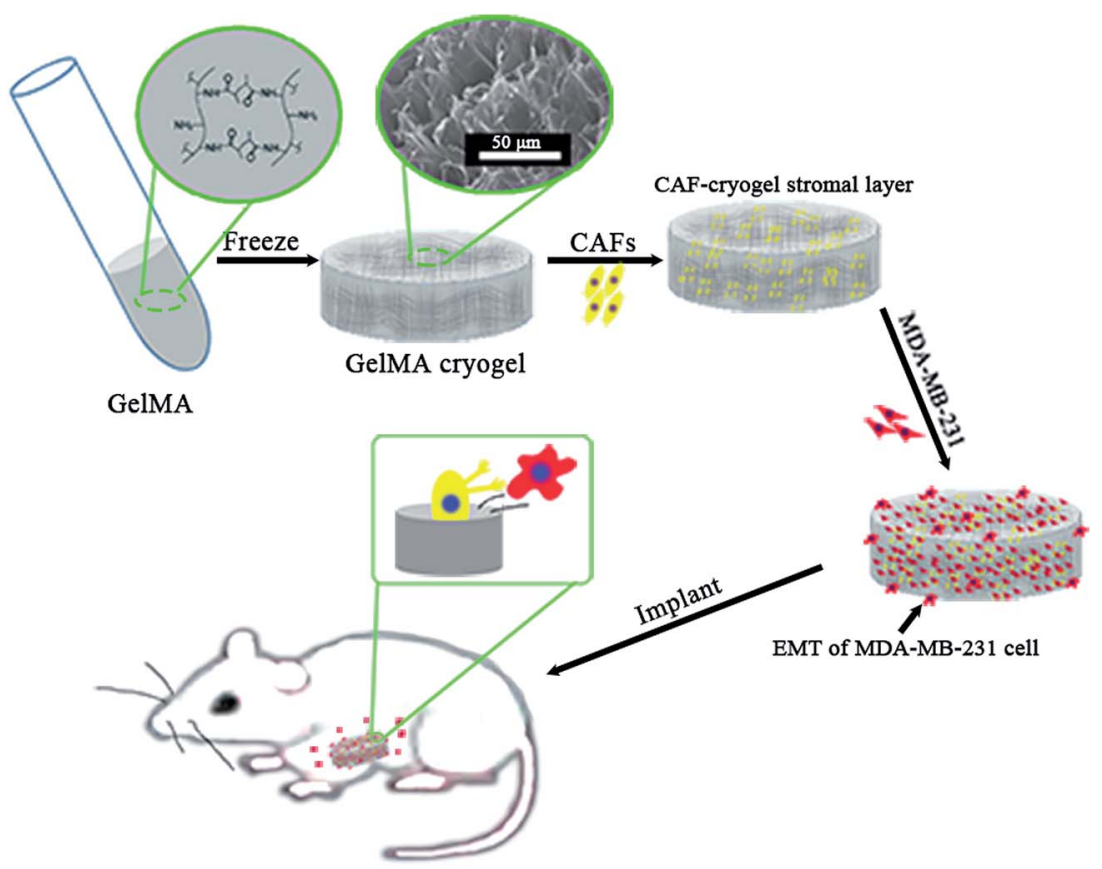

Scheme 1 The 3D co-culture system for the study of the role of CAFs in the EMT process of MDA-MB-231 cells in vitro and in vivo. 
of CAFs to breast cancer are elusive and complex, those methods may lead to unknown deviation. In the present research, the isolated CAFs were seeded straight on to the cryogel scaffolds to form a 3D stromal cell layer (Scheme 1). The cells isolated from the clinical human breast cancer samples grew well and were a spindle shape (Fig. 1A). The isolated cells displayed a positive expression of FAP which is the specific marker for fibroblasts (Fig. 1B). This indicated that most of the isolated cells were fibroblasts. ${ }^{25}$ In addition, the positive expression of $\alpha$-SMA proteins was detected in the isolated fibroblasts (Fig. 1C). This demonstrated that the isolated fibroblasts were CAFs rather than ordinary fibroblasts. ${ }^{26}$ The CAFs obtained were kept in a good condition, which ensured that all the subsequent experiments would proceed smoothly. The CAF-stromal cell layer provided a microenvironment for the human tumor that was more similar to that found in the human body.

\section{The cytocompatibility of the GelMA cryogel}

The characterization of the cryogel and the morphology of different cells co-cultured with cryogels were observed using SEM (Fig. 2). The newly developed GelMA cryogel displayed homogeneous microporous structures under SEM (Fig. 2A and A1). The cryogels exhibited relatively smooth and flat surfaces and different breast cancer cells adhered to the different orientation on the wall of the micropores (Fig. 2B, B1, C and C1). The pore sizes of the coarse cryogels were about $50 \mu \mathrm{m}$ (Fig. 2B and B1). However, when cultured with breast cells, the pore sizes of the cryogels were 100-200 $\mu \mathrm{m}$. A suitable pore size is necessary to promote cell proliferation in different cells. ${ }^{27-29}$ Because of the excellent elasticity of the GelMA cryogels, the pore size in the GelMA cryogels could be adjusted with the growth of the breast cancer cells. Different 3D cell morphology was exhibited in this type of 3D culture system, for example, MDA-MB-231 cells had a round shape, whereas MCF-7 cells had
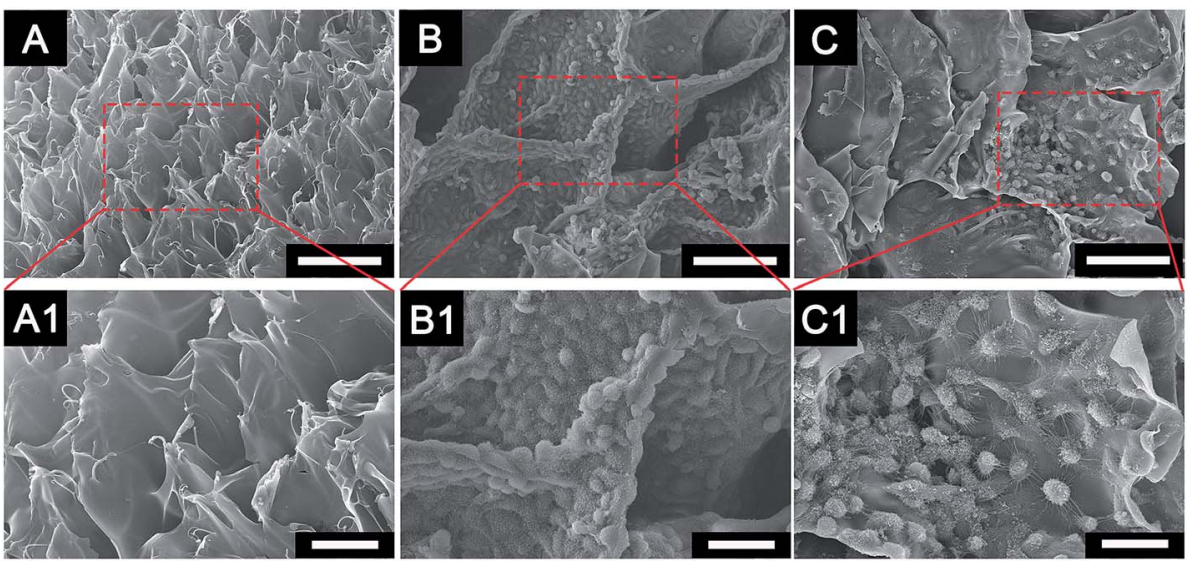

Fig. 2 Scanning electron microscopy of the cryogels and cell-seeded cryogels. (A) and (A1) The pure cryogel displayed a porous structures under SEM. (B) and (B1) The morphology of MDA-MB-231 cells cultured on the cryogel. (C) and (C1) The morphology of MCF-7 cells cultured on the cryogel. (Scale bar $=100 \mu \mathrm{m}$ in A, B and C. Scale bar $=50 \mu \mathrm{m}$ in A1, B1 and C1).
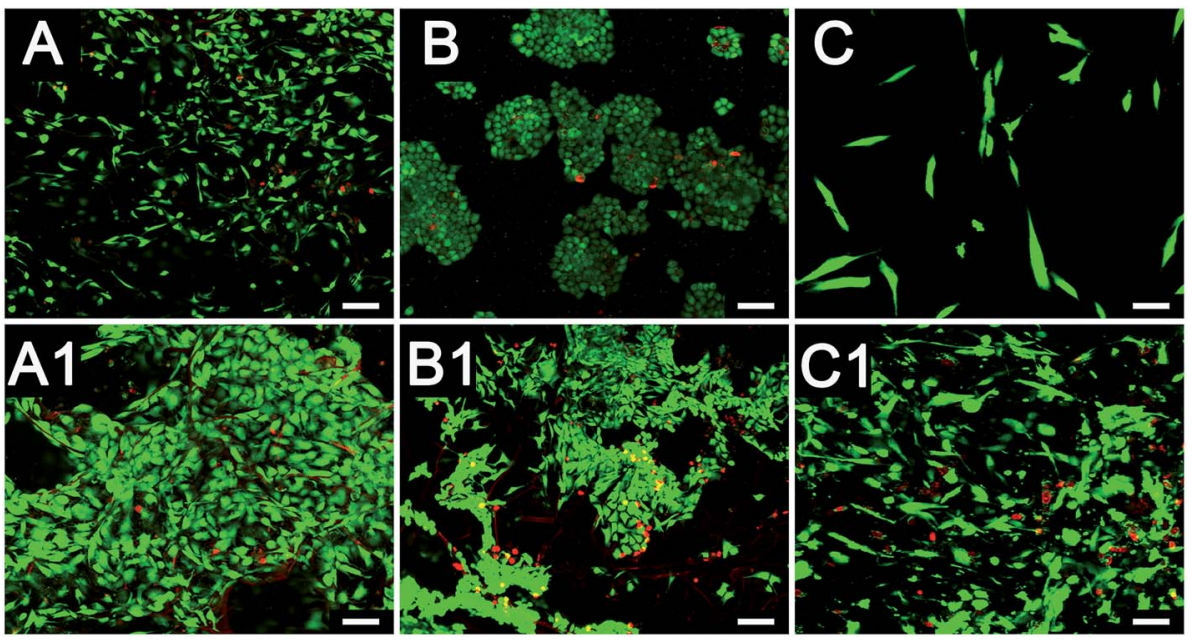

Fig. 3 Live/dead staining of the cryogels with MDA-MB-231 cells, MCF-7 cells and CAFs after culturing for three days. (A) and (A1) are the MDAMB-231 cells. (B) and (B1) were the MCF-7 cells. (C) and (C1) are the CAFs. (A), (B) and (C) are the 2D control groups. (A1-C1) are the groups that had different cells cultured on the cryogels. (Scale bars $=100 \mu \mathrm{m}$ ). 


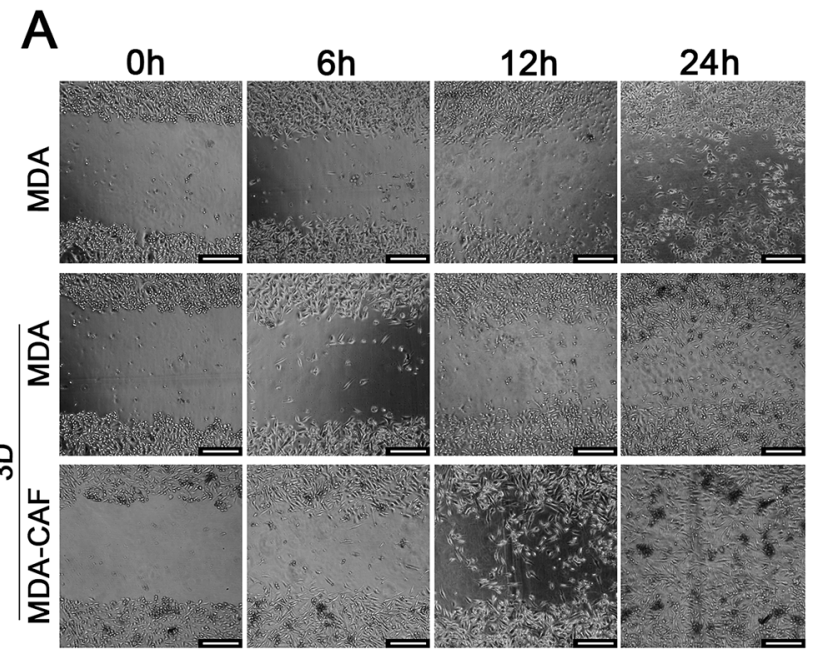

B
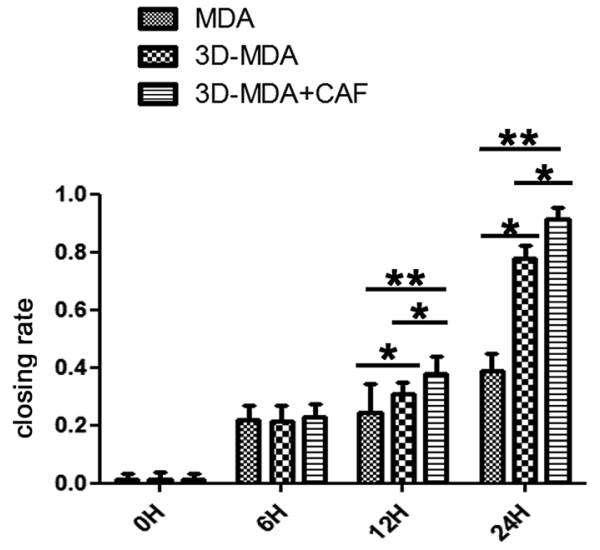

Fig. 4 The increased migration ability of the MDA-MB-231 cells was detected in the 3D-MDA/CAF group. (A) The wound healing assay of the cells in different groups (scale bars $=1000 \mu \mathrm{m}$ ). (B) The closing rate in the different groups. * and $* *$ are $p<0.05$ and $p<0.01$, respectively. several long pseudopodia and adhered to the scaffolds tightly. Thick and tenacious wall structures of the pores in the cryogel were generated by the interaction between the cells and the cryogels (Fig. 2B, B1, C and C1). Excellent biocompatibility of the cryogels was detected using live/dead staining by seeding different cells (MDA-MB-231 cells, MCF-7 cells and CAFs cells) on the 3D gels for three days (Fig. 3). Compared with the cells in the $2 \mathrm{D}$ culture system, the cells seeded on the cryogels grew much more intensively. Compared with the cells in the 2D culture, the MCF-7 cells in the 3D cryogel scaffold displayed a much more obvious spindle shape (Fig. 3B and B1). The MDAMB-231 cells were uniformly and crowdedly distributed on the scaffolds and showed good cell viability. Compared with that in the pure $2 \mathrm{D}$ culture system, more complicated cross-talking between cells would appear in the $3 \mathrm{D}$ co-cultue system. It was supposed that the interaction of the cells in the $3 \mathrm{D}$ co-culture system in vitro was similar to the microenvironment in vivo.

\section{CAFs promote EMT of MDA-MB-231 cells in 3D cryogel co- culture system in vitro}

Reports showed that the CAFs could induce the cancer cells to become invasive and metastasise, and the migration to distant sites is an important marker for the invasive and metastasis abilities of cancer cells. ${ }^{30,31}$ The scratch wound healing assay was carried out to determine whether co-cultured CAFs could affect the migration ability of the MDA-MB-231 cells. The 3D coculture system in which MDA-MB-231 cells were co-cultured with CAFs in the $3 \mathrm{D}$ cryogel scaffold was described as the $3 \mathrm{D}$ MDA/CAF group. The 3D co-culture system in which pure MDA-MB-231 cells were cultured in the 3D cryogel scaffold without the CAF stromal cell layer was described as the 3D-MDA group. The 2D culture system in which pure MDA-MB-231 cells were cultured in $2 \mathrm{D}$ plates was described as the control group (2D group). After being dissociated from the 3D-MDA/CAF coculture scaffolds, 3D-MDA culture scaffolds and the $2 \mathrm{D}$
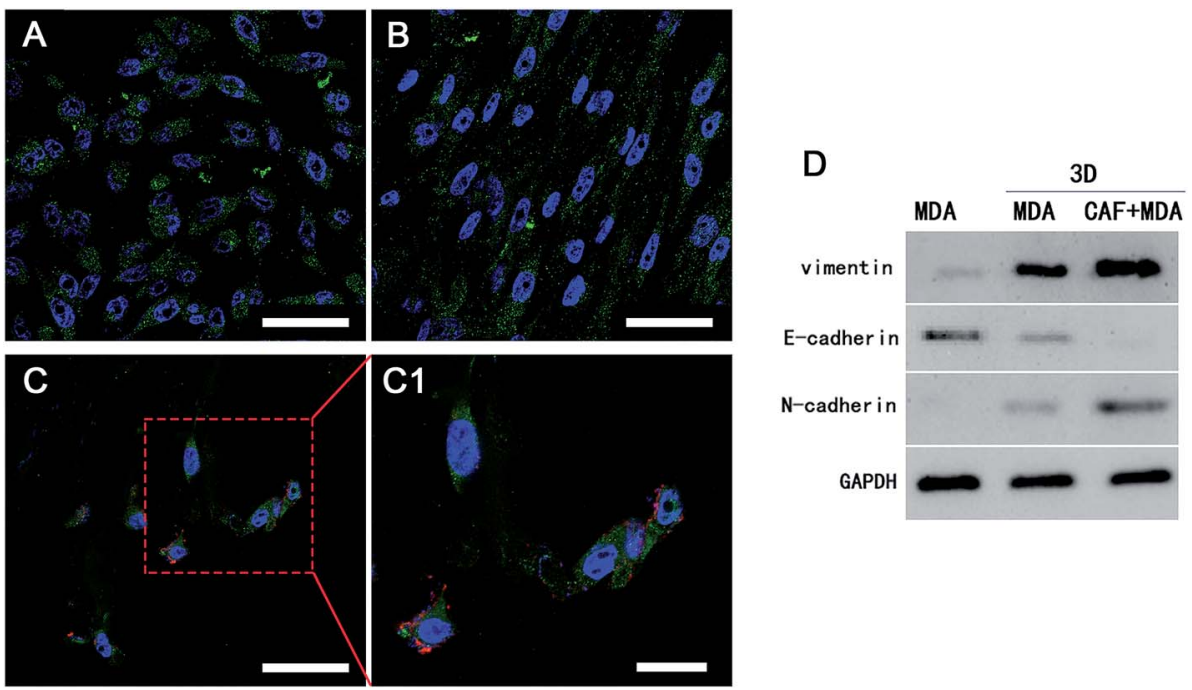

Fig. 5 The obvious cell EMT process was presented in the 3D-MDA/CAF group. (A) The 2D control group. (B) The 3D-MDA group. (C) and (C1) The 3D-MDA/CAFs group. Blue: DAPI, green: E-cadherin, red: Ki67. (Scale bar $=50 \mu \mathrm{m}$ in A-C. Scale bar $=20 \mu \mathrm{m}$ in C1). (D) Western blot analysis. 
culture plates, the cells were cultured on plates for $24 \mathrm{~h}$ and this was followed by the scratching of confluent monolayers on the cells grown on the plates. The cell migration abilities were determined using the ratio of the scratch wound healing area. As shown in Fig. 4A, a higher closing rate was displayed in both the 3D-MDA/CAF group and the 3D-MDA group compared with the $2 \mathrm{D}$ group after culturing for $12 \mathrm{~h}$. The closing rate was the highest in the 3D-MDA/CAF group out of all the three groups (Fig. 4B). These results supported the fact that the 3D-MDA/CAF group exhibited the highest migration ability out of the three groups, which meant that the breast cancer cells in this coculture system had better invasive and metastasis abilities. This also implied that the CAFs might promote MDA-MB-231 cell migration in the 3D cryogel co-culture system.
The invasive and metastasis abilities can be also verified by the initiation of EMT. EMT is a basic physiological and pathological phenomenon and is involved in embryonic development, organogenesis and tumor metastasis. ${ }^{32,33}$ The EMT is closely related to a malignant phenotype of tumors, which is marked by the reduction or loss in epithelial E-cadherin protein and the increasing expression of the interstitial N-cadherin protein. ${ }^{34}$ E-cadherin mediated cell-cell connections are an important element for maintaining the integrity and polarity of cells. ${ }^{35}$ The absence of E-cadherin destroys the adhesion between cells, which then leads to EMT and then enhances the invasion and metastasis of tumor cells, ${ }^{36,37}$ whereas N-cadherin mediates the enhancement of tumor cell invasion and metastasis. $^{38,39}$ The increasing expressions of $\mathrm{N}$-cadherin and
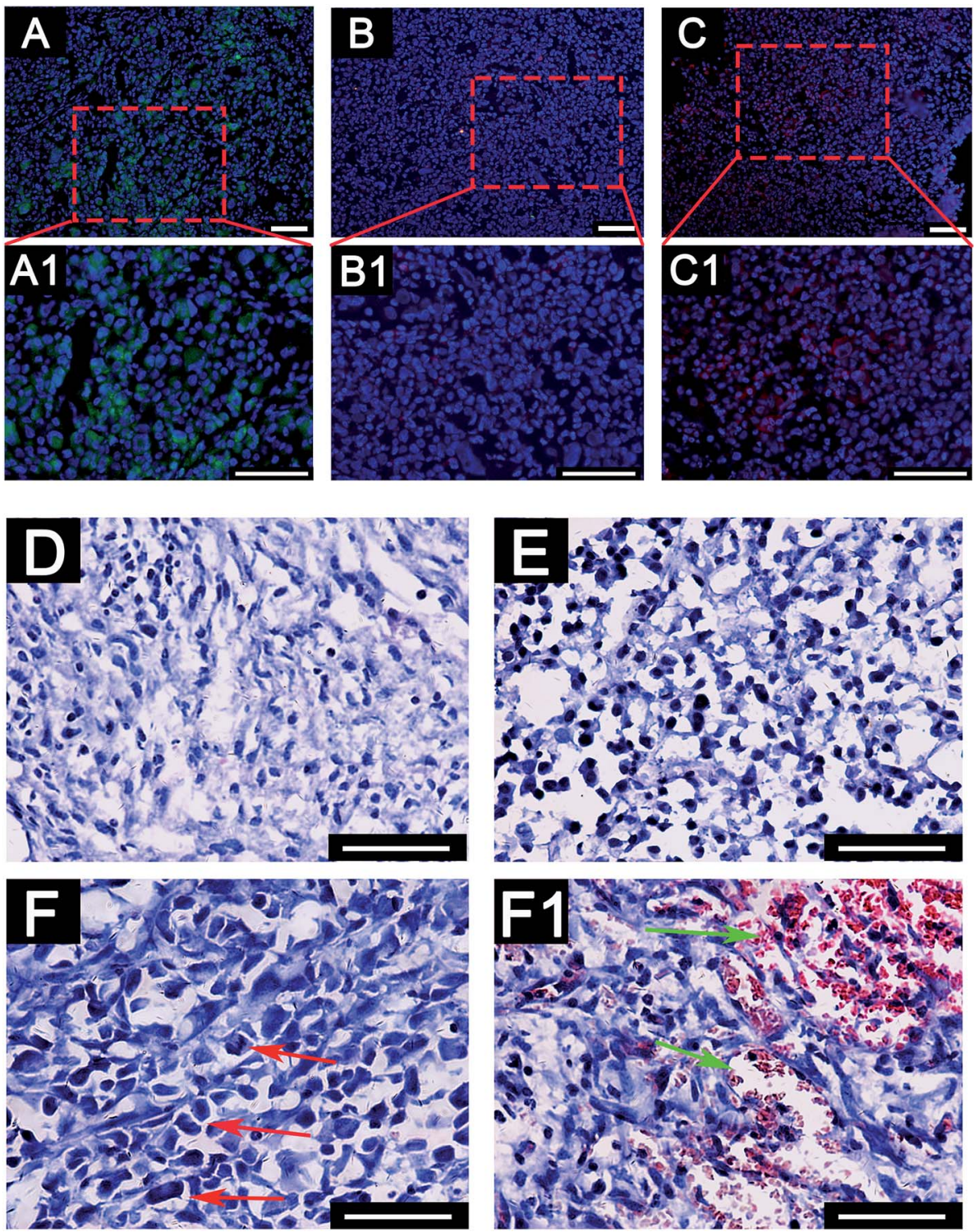

Fig. 6 Excessive deterioration and metastasis of MDA-MB-231 cells in the 3D cryogel system was detected in a xenograft breast tumor mouse model. (A-C1) The immunofluorescence assay of the tumor tissues. Green: E-cadherin proteins, red: N-cadherin proteins. (D-F1) The H\&E staining of the tumor tissues. (A), (A1) and (D) The 2D control groups. (B), (B1) and (E): The 3D-MDA group. (C), (C1), (F) and (F1) The 3D-MDA/CAFs group. Red arrows: atypical cells. Green arrows: new vessel growth (scale bars $=100 \mu \mathrm{m}$ ). 
vimentin proteins, accompanied by the loss of E-cadherin protein are well known markers of EMT. ${ }^{40}$ In agreement with a previous study, ${ }^{\mathbf{4 1}}$ a positive expression of Ki67 in the cytoplasm only appeared in the 3D-MDA/CAF group when immunofluorescence staining was used (Fig. 5A-C1) and this implied that CAFs could promote the EMT of MDA-MB-231 cells. Compared to those expressions of EMT-associated proteins in the $2 \mathrm{D}$ group and the 3D-MDA group, higher expressions of vimentin and $\mathrm{N}$ cadherin proteins and a lower expression of E-cadherin proteins were detected in the 3D-MDA/CAF group on day 3 (Fig. 5D). These results indicate that CAFs promoted EMT of MDA-MB231 cells in the $3 \mathrm{D}$ cryogel co-culture system in vitro.

\section{D-MDA/CAF co-culture hybrid exhibited high deterioration and metastasis in the xenograft breast tumor mouse model}

To further investigate the deterioration and metastasis of the 3D-MDA/CAF co-culture hybrid in vivo, the 3D-MDA/CAF coculture hybrid, the 3D-MDA culture hybrid and the 2Dcultured cells were transplanted into the axilla of separate nude mouse. In agreement with the results obtained in vitro, there was a significant increase in the number of $\mathrm{N}$-cadherin positive cells and a decreased number of E-cadherin positive cells in the primary tumor in the 3D-MDA/CAF hybrid transplanted group (Fig. 6A-C1). A few N-cadherin positive cells and a few E-cadherin positive cells were detected in the 3D-MDA hybrid transplanted group. Whereas E-cadherin proteins were highly expressed in the cells of the $2 \mathrm{D}$ group. H\&E staining showed that the tumor cells in the 3D-MDA/CAF group displayed an abnormal malignant tumor-specific nucleus (Fig. 6DF). As shown in Fig. 6, the nucleus of the cell in the 3D-MDA/CAF group was the biggest amongst the three groups, and a hypersegmented nucleus could be detected in the 3D-MDA/CAF group. Both the presence of heteromorphic nuclei and angiogenesis are the basic evidence for tumor deterioration. ${ }^{42}$ In the 3D-MDA/CAF group, a large amount of new vessels were detected in the tumor sites, which offered nutrition to the tumor cells and promoted its malignant migration. These results indicate that CAFs could promote deterioration of MDAMB-231 cells in a 3D cryogel co-culture system in vivo.

\section{Conclusion}

In conclusion, the GelMA cryogels are desirable scaffolds for the study of cell interaction between the breast cancer cells and stromal cells under 3D co-culture conditions. Using the 3D cryogel co-culture system developed in this research, it was found that CAFs could promote the EMT of MDA-MB-231 cells in vitro and promote deterioration and metastasis of MDA-MB231 cells in vivo. This simple 3D cryogel co-culture system may be a convenient approach for studying the EMT mechanism and a useful platform for anti-cancer drug screening in vitro.

\section{Acknowledgements}

This work was supported by the National Natural Science Foundation of China (31572343, 31371000, and 51428301).

\section{References}

1 Y. Mao, E. T. Keller, D. H. Garfield, K. Shen and J. Wang, Cancer Metastasis Rev., 2013, 32, 303-315.

2 T. S. Xia, J. Wang, H. Yin, Q. Ding, Y. F. Zhang, H. W. Yang, X. A. Liu, M. Dong, Q. Du and L. J. Ling, Oncol. Rep., 2010, 24, 203-211.

3 A. Orimo, P. B. Gupta, D. C. Sgroi, F. Arenzana-Seisdedos, T. Delaunay, R. Naeem, V. J. Carey, A. L. Richardson and R. A. Weinberg, Cell, 2005, 121, 335-348.

4 O. E. Franco, A. K. Shaw, D. W. Strand and S. W. Hayward, Semin. Cell Dev. Biol., 2010, 21, 33-39.

5 P. J. Mishra, P. J. Mishra, R. Humeniuk, D. J. Medina, G. Alexe, J. P. Mesirov, S. Ganesan, J. W. Glod and D. Banerjee, Cancer Res., 2008, 68, 4331-4339.

6 A. Orimo and R. A. Weinberg, Cell Cycle, 2006, 5, 1597-1601. 7 P. S. Soon, E. Kim, C. K. Pon, A. J. Gill, K. Moore, A. J. Spillane, D. E. Benn and R. C. Baxter, Endocr.-Relat. Cancer, 2012, 20, 1-12.

8 S. Y. Park, H. M. Kim and J. S. Koo, Breast Cancer Res. Treat., 2015, 149, 727-741.

9 K. Yue, G. Trujillo-de Santiago, M. M. Alvarez, A. Tamayol, N. Annabi and A. Khademhosseini, Biomaterials, 2015, 73, 254-271.

10 K. Tekin, L. Uzun, Ç. A. Şahin, S. Bektaş and A. Denizli, React. Funct. Polym., 2011, 71, 985-993.

11 I. Inci, H. Kirsebom, I. Y. Galaev, B. Mattiasson and E. Piskin, J. Tissue Eng. Regener. Med., 2013, 7, 584-588.

12 S. T. Koshy, T. C. Ferrante, S. A. Lewin and D. J. Mooney, Biomaterials, 2014, 35, 2477.

13 Z. Munoz, H. Shih and C. C. Lin, Biomater. Sci., 2014, 2, 1063-1072.

14 S. J. Hollister, Nat. Mater., 2005, 4, 518-524.

15 B. S. Kim and D. J. Mooney, Trends Biotechnol., 1998, 16, 224230.

16 Y. C. Chiu, H. Engel, J. C. Larson, M. H. Cheng, S. W. Kao, S. Gupta and E. M. Brey, Biomaterials, 2011, 32, 6045-6051.

17 L. Wang, J. Jiang, W. Hua, A. Darabi, X. Song, C. Song, W. Zhong, M. M. Q. Xing and X. Qiu, Adv. Funct. Mater., 2016, 26, 4293-4305.

18 S. Paget, Cancer Metastasis Rev., 1989, 8, 98-101.

19 F. Spill, D. S. Reynolds, R. D. Kamm and M. H. Zaman, Curr. Opin. Biotechnol., 2016, 40, 41-48.

20 G. Balachander, S. Balaji, A. Rangarajan and K. Chatterjee, ACS Appl. Mater. Interfaces, 2015, 7, 27810-27822.

21 J. Liu, Y. Tan, H. Zhang, Y. Zhang, P. Xu, J. Chen, Y. C. Poh, K. Tang, N. Wang and B. Huang, Nat. Mater., 2012, 11, 734741.

22 S. Florczyk, F. Kievit, K. Wang, A. Erickson, R. Ellenbogen and M. Zhang, J. Mater. Chem., 2016, 4, 6326-6334.

23 R. Kalluri and M. Zeisberg, Nat. Rev. Cancer, 2006, 6, 392401.

24 A. Ostman and M. Augsten, Curr. Opin. Genet. Dev., 2009, 19, 67-73.

25 M. Loeffler, J. A. Krüger, A. G. Niethammer and R. A. Reisfeld, J. Clin. Invest., 2009, 116, 1955-1962. 
26 N. Erez, M. Truitt, P. Olson and D. Hanahan, Cancer Cell, 2010, 17, 135-147.

27 B. B. Mandal and S. C. Kundu, Biomaterials, 2009, 30, 29562965.

28 S. M. Lien, L. Y. Ko and T. J. Huang, Acta Biomater., 2009, 5, 670-679.

29 S. H. Oh, T. H. Kim, G. I. Im and J. H. Lee, Biomacromolecules, 2010, 11, 1948-1955.

30 Y. Yu, C. H. Xiao, L. D. Tan, Q. S. Wang, X. Q. Li and Y. M. Feng, Br. J. Cancer, 2014, 110, 724-732.

31 R. A. Saito, P. Micke, J. Paulsson, M. Augsten, C. Peña, P. Jönsson, J. Botling, K. Edlund, L. Johansson and P. Carlsson, Cancer Res., 2010, 70, 2644-2654.

32 K. Vleminckx, L. Vakaet Jr, M. Mareel, W. Fiers and R. F. Van, Cell, 1991, 66, 107-119.

33 R. B. Hazan, A. G. R. Phillips, A. R. F. Qiao, A. L. Norton and S. A. A. Aaronson, J. Cell Biol., 2000, 148, 779-790.

34 H. Acloque, M. S. Adams, K. Fishwick, M. Bronner-Fraser and M. A. Nieto, J. Clin. Invest., 2009, 119, 1438-1449.
35 H. Oka, H. Shiozaki, K. Kobayashi, M. Inoue, H. Tahara, T. Kobayashi, Y. Takatsuka, N. Matsuyoshi, S. Hirano and M. Takeichi, Cancer Res., 1993, 53, 1696-1701.

36 B. Kocic, S. Filipovic, B. Petrovic, D. Mijalkovic, N. Rancic and A. Poultsidi, Journal of Balkan Union of Oncology, 2010, 15, 660-667.

37 J. Chen, N. Imanaka, J. Chen and J. D. Griffin, Br. J. Cancer, 2010, 102, 351-360.

38 S. Nakajima, R. Doi, E. Toyoda, S. Tsuji, M. Wada, M. Koizumi, S. S. Tulachan, D. Ito, K. Kami and T. Mori, Clin. Cancer Res., 2004, 10, 4125-4133.

39 M. T. Nieman, R. S. Prudoff, K. R. Johnson and M. J. Wheelock, J. Cell Biol., 1999, 147, 631-644.

40 F. T. Martin, R. M. Dwyer, J. Kelly, S. Khan, J. M. Murphy, C. Curran, N. Miller, E. Hennessy, P. Dockery and F. P. Barry, Breast Cancer Res. Treat., 2010, 124, 317-326.

41 D. Faratian, A. Munro, C. Twelves and J. M. Bartlett, Histopathology, 2009, 54, 254-257.

42 Y. Honda, T. Kawaoka, H. Aikata, H. Kan, H. Fujino, T. Kobayashi, T. Fukuhara, N. Naeshiro, A. Hiramatsu and M. Imamura, Hepatol. Res., 2014, 45, 705-710. 